\title{
On the origin of pre-reversal enhancement of the zonal equatorial electric field
}

\author{
M. C. Kelley ${ }^{1}$, R. R. IIma ${ }^{1}$, and G. Crowley ${ }^{2}$ \\ ${ }^{1}$ School of Electrical and Computer Engineering, Cornell University, Ithaca, NY, USA \\ ${ }^{2}$ Atmospheric \& Space Technology Research Associates (ASTRA), San Antonio, TX, USA
}

Received: 1 October 2008 - Revised: 26 February 2009 - Accepted: 9 April 2009 - Published: 5 May 2009

\begin{abstract}
In November 2004, a large and variable interplanetary electric field (IEF) was felt in the reference frame of the Earth. This electric field penetrated to the magnetic equator and, when the Jicamarca Radio Observatory (JRO) was in the dusk sector, resulted in a reversal of the normal zonal component of the field. In turn, this caused a counter-electrojet (CEJ), a westward current rather than the usual eastward current. At the time of the normal pre-reversal enhancement (PRE) of the eastward field, the Jicamarca incoherent scatter radar (ISR) observed that the westward component became even more westward. Two of the three current explanations for the PRE depend on the neutral wind patterns. However, this unique event was such that the neutral wind-driven dynamos could not have changed. The implication is that the Haerendel-Eccles mechanism, which involves partial closure of the equatorial electrojet (EEJ) after sunset, must be the dominant mechanism for the PRE.
\end{abstract}

Keywords. Ionosphere (Equatorial ionosphere; Ionospheric irregularities; Modeling and forecasting)

\section{Introduction}

One of the most important features of the equatorial ionosphere is the PRE of the zonal electric field. This phenomenon causes a brief and intense uplift of the electric field near sunset, which results in a height increase in the equatorial ionosphere and an increase in the growth rate of the generalized Rayleigh-Taylor instability. This effect has been studied primarily at one location - Jicamarca, Peru - for over 40 years, and its seasonal and solar cycle patterns have been established.

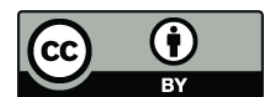

Correspondence to: M. C. Kelley (mikek@ece.cornell.edu)
At present, three competing theories exist for the PRE, which we consider here in light of a unique application for a large electric field of interplanetary origin.

\section{Data presentation}

In November 2004, a magnetic "superstorm" occurred while a "World Day" incoherent scatter run was underway (Kelley et al., 2009b). The associated changes in the interplanetary magnetic field (IMF) created electric fields of huge proportions in the rest frame of the earth, over $30.0 \mathrm{~V} / \mathrm{km}$, and 3.0 MV across the magnetosphere. Figure 1 shows the dawnto-dusk component of the IEF divided by ten, along with the observed zonal electric field over Jicamarca.

These rapid and early variations in the IEF are pure in the sense that the other high latitude effect, the disturbance dynamo, cannot develop on this time scale. As shown in Fig. 2, a run of the TIMEGCM model confirms that, at sunset on 9 November, the zonal equatorial winds were of normal sign and actually lower than normal. The other two components show only gravity wave activity. This is verified in Fig. 3, which shows only gravity waves, no large mean winds at Arecibo. A prompt-penetrating electric field (PPE) can only be in effect at the equator well into the evening.

The large southward IMF between 18:00 and 21:00 UT on 9 November occurred when Peru was on the dayside. The EEJ, of course, increased in magnitude but, surprisingly, not by as much as the electric field. This suggests that plasma waves create an anomalous resistivity, which is discussed elsewhere (Kelley et al., 2009a). Of import for our purposes is that the IMF reversed sign, creating what is called a CEJ. Ground magnetometer data are difficult to interpret since a substantial ring current was flowing. To our relief, the CHAMP (CHAllenging Minisatellite Payload) magnetometer detector can cope with such effects and confirmed that a CEJ occurred (P. Alken, personal communication, 2008).

Published by Copernicus Publications on behalf of the European Geosciences Union. 


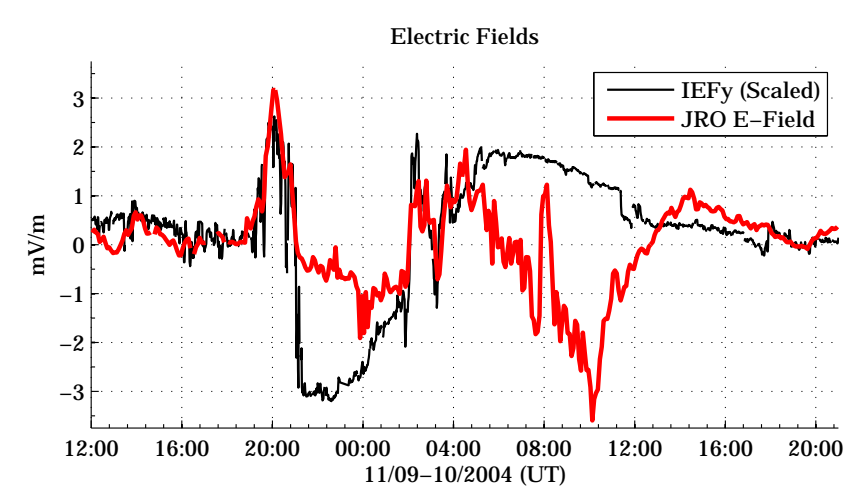

Fig. 1. The black-line plot is the y-component of the electric field in the Earth's frame of rest delayed by the transit time of the solar wind to the front of the magnetosphere divided by ten for a 33-h period in November 2004. The red-line plot is the zonal component of the electric field and vertical drift in the equatorial ionosphere ( $3 \mathrm{mV} / \mathrm{m}$ corresponds to $120 \mathrm{~m} / \mathrm{s}$ ). The local time in Peru is UT-5.

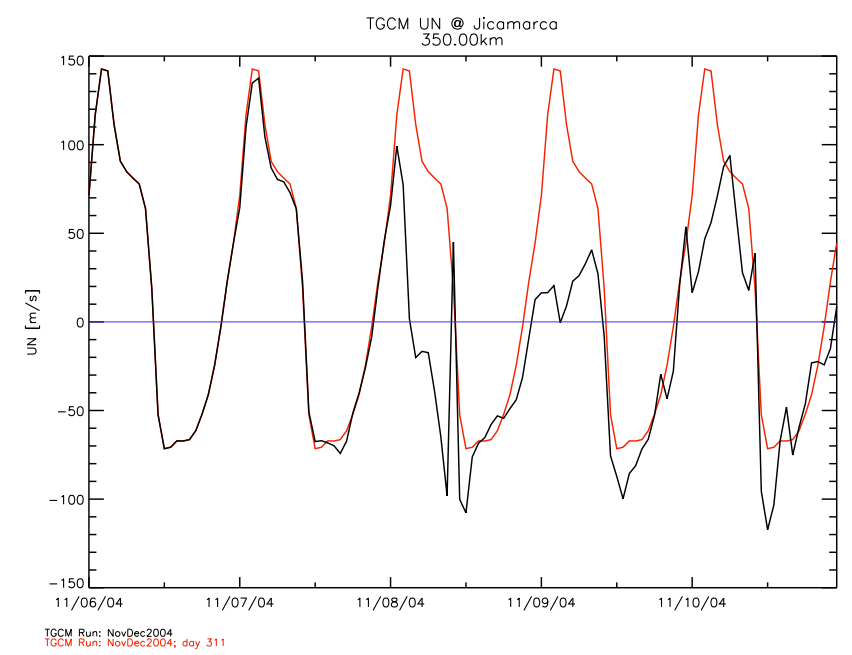

Fig. 2. TIMEGCM model run of the zonal wind over Peru for five days in November 2004. 6 November was quiet and has been reproduced with every day in red for comparison. UN was actually small near local sunset on 9 November (00:00 UT) but remained eastward.

The important issue here is that, near sunset, the EEJ was reversed.

This data set has been used to drive the AFRL Convective Equatorial Ionospheric Storms model with some success (Kelley and Retterer, 2008). The model was driven by the IEF divided by 10 for $B_{z}$ pointing to the south, by 33 for $B_{z}$ north, and by a change of sign after 23:00 LT. This field was superposed on the quiet time Scherliess-Fejer field model (Scherliess and Fejer, 1999). The results are presented in Fig. 4. Although one can quibble with these linear relationships, the two measurements are $85 \%$ correlated using this model. The "departure" after 23:00 LT is actually a local time dependence of the penetrating field (Kelley and Dao, 2009).
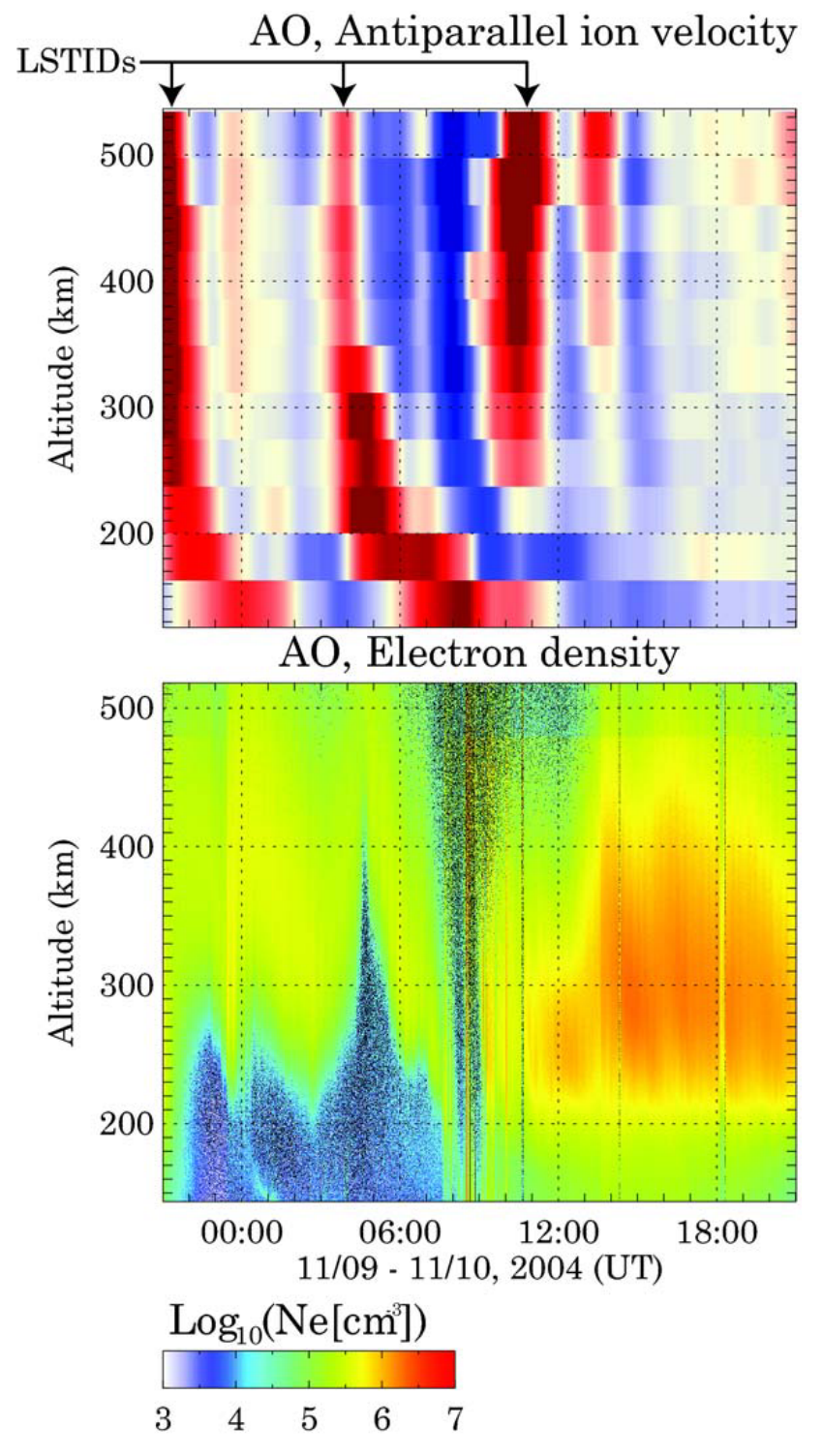

Fig. 3. Anti-parallel drifts and electron density over Arecibo. The arrows indicate large scale TIDs (adapted from Kelley et al., 2009b).

What caught our attention is the fact that, at the time when a normal pre-reversal enhancement was predicted by the quiet time model, the data showed a negative excursion, nearly a mirror image of the normal PRE.

\section{Discussion}

Three models exist for the PRE. In the Farley-Bonelli model (Farley et al., 1986), an eastward neutral wind blowing across the sunset terminator, coupled with a gradient in the conductivity locally and on the ends of the conjugate field lines, results in an extra positive charge layer. The electric field associated with this layer is eastward on the western edge and westward on the eastern edge. Since we have argued that the 


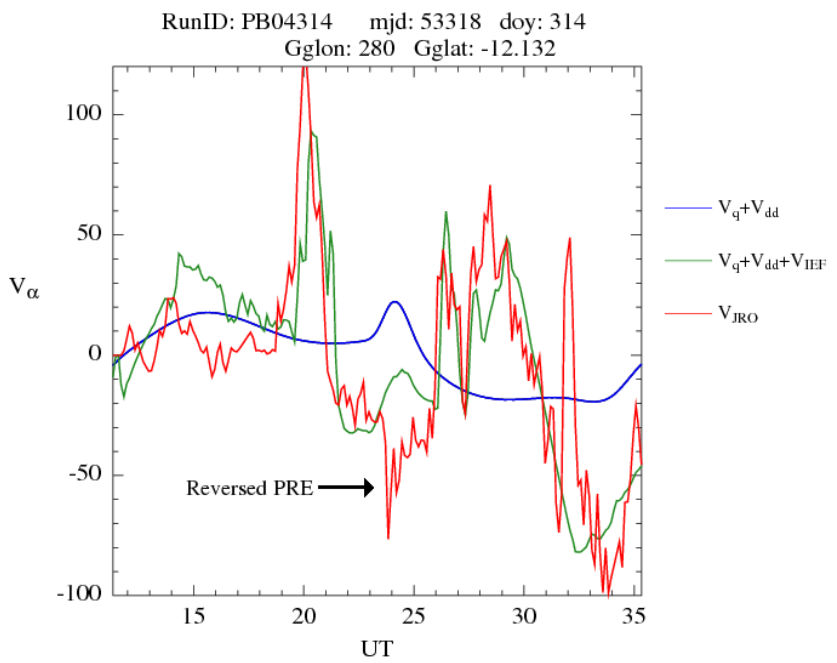

Fig. 4. The red line is the measured vertical velocity over the equator and the blue line is the quiet time velocity for the period. The green line is the predicted velocity based on the IEF (adapted from Kelley and Retterer, 2008).

wind field cannot yet be affected by the magnetic storm, this mechanism should be operating in the normal manner.

The second mechanism may be called the Rishbeth-Eccles model (Rishbeth, 1971; Eccles, 1998). This approach also relies on the eastward neutral wind and the fact that the F-region dynamo results in an increase in the downwarddirected electric field after sunset. The requirement that $\nabla \times \mathbf{E}=0$ then requires a corresponding change in the zonal electric field. The figures in the literature illustrating this effect are a bit confusing and hence a simple model has been created to illustrate the effect. Let the vertical electric field be of the form,

$E_{z}=-E_{0 z} \sin (m z) \tanh (k x)$

where $E_{0 z}$ is the maximum value of the vertical electric field, $m$ is the plasma shear's wavenumber, and $k$ is the wavenumber of the local time component. The $\tanh (k x)$ form models the fact that $E_{z}$ changes sign with local time. The $\sin (m z)$ form corresponds to the shear in the plasma flow. Our goal is to determine the zonal electric field consistent with this form and with $\nabla \times \mathbf{E}=0$. The components are related by,

$\frac{\partial E_{z}}{\partial x}=-E_{0 z} \sin (m z) \frac{d[\tanh (k x)]}{d x}=\frac{\partial E_{x}}{\partial z}$

Then, integrating with respect to $z$,

$E_{x}=E_{0 z} \frac{k}{m} \cos (m z)\left[1-\tanh ^{2}(k x)\right]+K$

where $K$ is a zonal electric field due to contributions from an unknown source. Equations (1) and (2) are plotted in Fig. 5 for which the following parameter values were utilized: $E_{0 z}=4.0 \mathrm{mV} / \mathrm{m}, k=2 \pi \cdot 0.001 \mathrm{~km}^{-1}, m=2 \cdot 0.01 \mathrm{~km}^{-1}$,
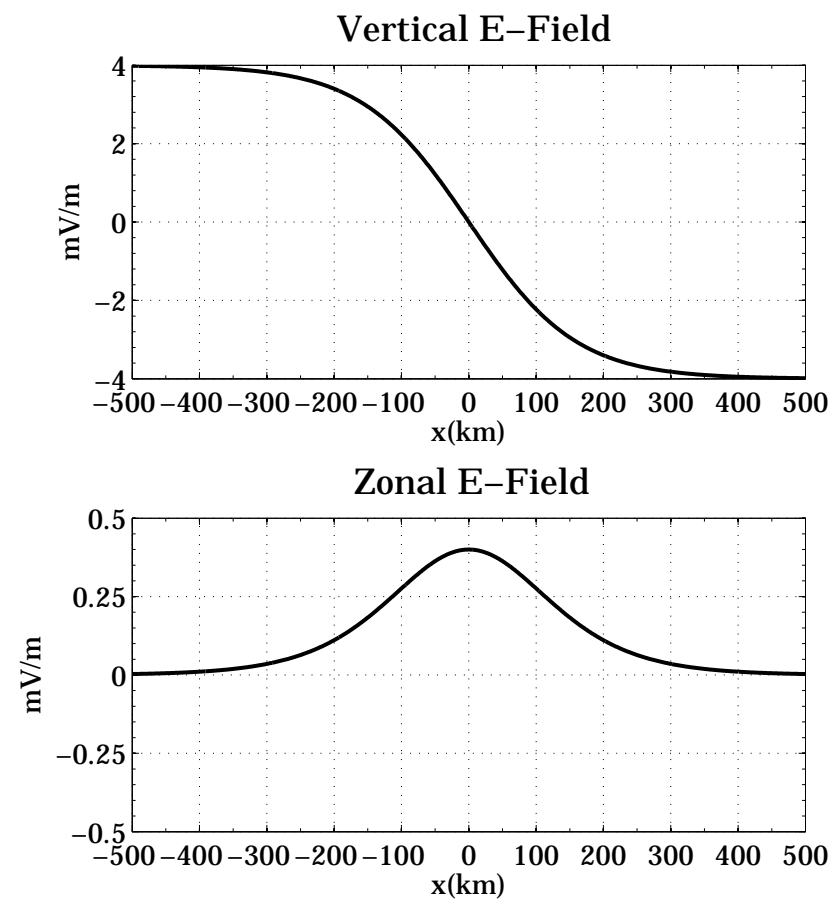

Fig. 5. Zonal electric field (bottom panel) required by $\nabla \times \mathbf{E}=0$, given the two-dimensional behavior of the vertical field component (top panel) near sunset.

$K=0.0 \mathrm{mV} / \mathrm{m}$, and $z=0.0 \mathrm{~km}$. The values of the $\mathrm{x}$-axes are in side the longitudinal interval of $\pm 5^{\circ}$ (equivalent to $\pm 500 \mathrm{~km}$ ) around the east-west direction with respect to the Jicamarca radar location. Figure 5 shows clearly the PRE effect caused by the special dependence of the zonal plasma flow.

The third mechanism is the Haerendel-Eccles model (Haerendel and Eccles, 1992), in which the EEJ partially closes in the post-sunset $\mathrm{F}$ region. The associated current system requires both a vertically upward electric field and an eastward zonal component in the post-sunset period.

With no likely change in the zonal wind pattern, both the Farley-Bonelli and the Rishbeth-Eccles mechanisms predict a normal eastward PRE for this night. The fact that an inverted PRE occurred during CEJ conditions thus seems to indicate that not only is the Haerendel-Eccles mechanism occurring, but it also overcomes both of the other processes.

\section{Conclusion}

The implication of this event is that the normal PRE must be created by EEJ current closure and the other two mechanisms are secondary.

Acknowledgements. This research was supported by the Atmospheric Science Section of the National Science Foundation under grant ATM-0551107.

Topical Editor K. Kauristie thanks G. Haerendel and two other anonymous referees for their help in evaluating this paper. 


\section{References}

Eccles, V.: Modeling investigation of the evening pre-reversal enhancement of the zonal electric field in the equatorial ionosphere, J. Geophys. Res., 103, 26709-26719, 1998.

Farley, D. T., Bonelli, E., Fejer, B. G., and Larsen, M. F.: The prereversal enhancement of the zonal electric field in the equatorial ionosphere, J. Geophys. Res., 91, 13723-13728, 1986.

Haerendel, G. and Eccles, J. V.: The role of the equatorial electrojet in the evening ionosphere, J. Geophys. Res., 97, 1181-1197, 1992.

Kelley, M. C. and Dao, E.: On the local time dependence of the penetration of solar wind-induced electric fields to the magnetic equator, Ann. Geophys., in press, 2009.
Kelley, M. C. and Retterer, J. M.: First successful prediction of a convective equatorial ionospheric storm using solar wind parameters, Space Weather, 6, S08003, doi:10.1029/2007SW000381, 2008.

Kelley, M. C., Ilma, R. R., Alken, P., and Maus, S.: Evidence for anomalous Cowling conductivity in the strongly driven equatorial electrojet and a reconciliation of magnetic field data with theory, Geophys. Res. Lett., submitted, 2009a.

Kelley, M. C., Ilma, R. R., Nicolls, M., Ericson, P., Goncharenko, L., Chau, J., and Aponte, N.: Spectacular low and mid-latitude electrical fields and neutral winds during a superstorm, J. Atmos. Solar-Terr. Phys., in press, 2009b.

Rishbeth, H.: Polarization fields produced by winds in the equatorial F-region, Planet. Space Sci., 19, 357-369, 1971.

Scherliess, L. and Fejer, B. G.: Radar and satellite global equatorial F region vertical drift model, J. Geophys. Res., 104, 6829-6842, 1999. 